\title{
Design and Evaluate A Model of Vehicle Suspension System By Using Fem
}

\author{
Vikesh Kumar Ranjan \\ M. Tech. Scholar \\ NRI Institute of Information Science and Technology \\ Bhopal, India \\ kvranjan95@gmail.com
}

\author{
Arun Patel \\ Professor \\ NRI Institute of Information Science and Technology \\ Bhopal, India \\ arunpatel83@gmail.com
}

\begin{abstract}
The suspension system assists the vehicle's braking system in terms of safety, driving pleasure and passenger comfort when driving without road noise, bumps or vibrations. If the roads were perfectly smooth, a vehicle suspension system would not be necessary. As a result, the wheel of the machine undergoes a sudden vertical movement as it moves over the bumps. To improve the stability of the suspension system design multiple spring in vehicle suspension system and the design multiple spring in vehicle suspension system for different materials to enhance the results. Final conclusion of the analysis the Titanium Ti-6Al-4V is the much batter material than other two materials Cast iron and titanium Ti13V-11Cr-3Al. Because the yield stress of Titanium Ti-6Al-4V is higher the titanium Ti-13V-11Cr-3Al. And the density of the Titanium Ti-6Al-4V was lower than cast iron and titanium Ti-13V-11Cr-3Al.
\end{abstract}

Keywords: FEA, ANYSIS, CATIA, Aluminum, cast iron, Titanium.

\section{INTRODUCTION}

The suspension system assists the vehicle's braking system in terms of safety, driving pleasure and passenger comfort when driving without road noise, bumps or vibrations. If the roads were perfectly smooth, a vehicle suspension system would not be necessary. It is these irregularities that disturb the wheels. Bumps and holes in the road raise and lower the wheel of a vehicle perpendicular to the road surface. The strength of force depends on whether the wheel encounters a weak or significant inequality. the wheel of the machine undergoes a sudden vertical movement as it moves over the bumps. Learn more about suspension design for light and low power vehicles that resist fatigue during operation. The suspension system includes the spring and the fluid damping system. The spring absorbs the energy of an applied force. This energy is stored in the spring until the force is released, so that the spring returns to its original size, shape and position. It is therefore important that the vehicle suspension keeps the wheel in contact with the road surface.

\section{1) Vehicle Engine and Suspension}

In a typical motor vehicle, the main competition lies between the manufacturing processes of the powertrain components and the suspension system. Typical forged components used in vehicles include the crankshaft, connecting rod, and camshaft and suspension components, such as the control arm, steering joint and wheel hub. For a better understanding of the vehicle components, we will briefly discuss here the technical characteristics of the engine and chassis components, in particular the steering joint.

\section{LITERATURE REVIEW}

M. M. Patunkar et al. [1] This article shows that leaf springs area unit one in every of the oldest suspension components that they still use frequently, especially in commercial vehicles. The literature review shows that leaf springs area unit designed as generalized force components, within which the position, speed and orientation of the axis bearings offer the reaction forces within the frame mounting positions. Another half should be targeted, as the automotive industry is increasingly interested in replacement the steel spring with a composite spring due to its high strength to weight ratio.

B. Pyttel et al. [2] this text presents long-term fatigue tests on compressed helical compression springs, that were created using a special springloaded $40 \mathrm{~Hz}$ fatigue tester. The test springs were composed of 3 totally different materials: hardened spring valve steel oil and steel for valve springs in $\mathrm{SiCrV}$ alloy and stainless-steel. With a selected test strategy in a very test cycle, up to 500 springs with a wire diameter of $\mathrm{d}=3.0$ millimeter or 900 springs with $\mathrm{d}=1.6$ metric linear unit were tested at completely different stress levels simultaneously. 
Yunan Prawoto et al. [3] This article describes the helical springs of the vehicle, their distribution of basic stresses, the properties of the fabric, the producing and therefore the common defects. a detailed discussion of the parameters that influence the quality of the volute springs is also presented. The coil springs are not excluded, that corresponds to the tendency of the automotive industry to constantly reduce weight. A consequence of the load reduction effort is that the need to use elastic device materials with considerably greater efforts decades a gone than similar styles. The use of a superior steel strength has each benefits and disadvantages.

Ladislav Kosec et al. [4] during this document, the failure analysis of a spring of a vehicle of a rear shock absorber is examined and analyzed. Since the protecting layer on the surface of the spring is damaged, corrosion has occurred. the mix of corrosion and fatigue has resulted within the failure of a helical spring for cars.

\section{OBJECTIVE}

- To investigate the vehicle suspension system by improving finite element analysis (FEA)

- To analysis and compare von misses stresses and factor of safety by the previous material.

- To improve the stability of the suspension system design multiple spring in vehicle suspension system

- To analysis the design multiple spring in vehicle suspension system for different materials to enhance the results.

\section{METHODOLOGY}

\section{1) FEA Analysis on Vehicle Suspension System.}

In this project, we use the CAD software and ANSYS version 18.2 analysis. Here we can create a CAD model and determine the stress value and the deformation value in ANSYS. 18.2 To study different constraints and deformations, these were imported into the ANSYS network software and gave different results. The mesh model is essentially made up of nodes and elements. Tetra elements offer a better result than other types of elastic elements. Therefore, the elements used in this analysis are gloomy elements. The calculated forces and boundary conditions were applied to the mesh model in ANSYS 18.2. The design parameters obtained from the analysis of the finite elements above were compared for the materials and the best was selected.

\subsection{Preparation of model}

A CAD model is prepared in CATIA.

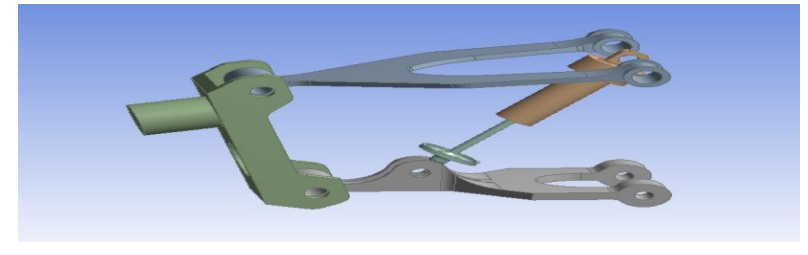

Figure 1: CFD model is prepared in CATIA.

\section{2) Steps of Working}

Step 1: Collecting information and data related to VSS.

Step 2: A fully parametric model of the VSS for both cases are created in CATIA V5R20

Step 3: Model obtained in Step 2 is analyzed using ANSYS 18.2.

Step 4: Finally, we compare the results obtained from ANSYS.

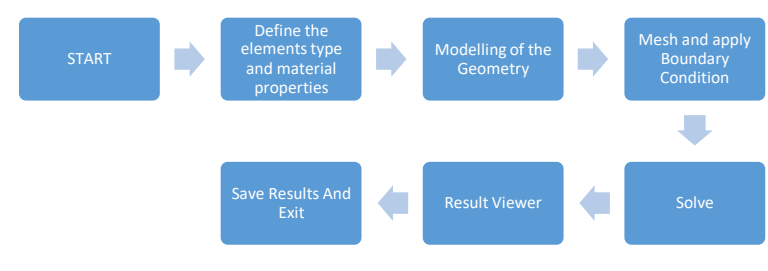

Figure 2: Setup of working

\section{3) Finite elements analysis}

Finite element analysis is a branch of fluid mechanics that uses numerical analysis and data structures to solve and analyze problems involving fixed structures. The ANSYS 18.2 software was used for this work.

\section{ANSYS functions:}

Finite element analysis uses the ANSYS software that engineers can use to perform the following tasks:

- To create prototypes and computer components, transfer the CAD models of structures into a system product.

- Improves the profile of structural elements through the optimization of the shape.

- It is possible to study physical reactions such as voltage levels, temperature distributions or electromagnetic fields.

- To reduce production costs, design optimization occurs at the beginning of the development process. 
- Test prototypes in environments where this would otherwise be undesirable or impossible (eg biomedical applications).

\section{4) Steps of ANSYS Analysis}

The different analysis steps involved in ANSYS are mentioned below.

\section{Preprocessor}

The model setup is basically done in preprocessor. The different steps in pre-processing are

- $\quad$ Build the model

- Define materials

- Generation of element mesh

2. Building The Model

- Creating a solid model within Catia.

- Importing model created in a computer-aided design (CAD) system.

\section{Case-1 Design Model for $1^{\text {st }}$ Case.}

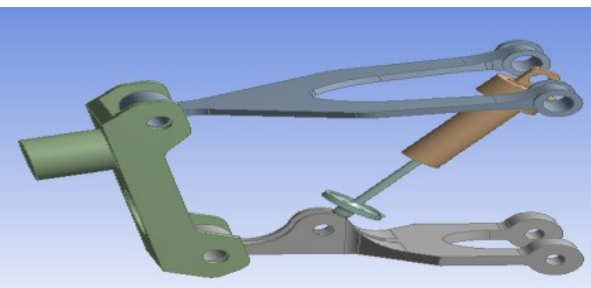

Figure 3: CAD model prepared in CATIA (base paper model)

CASE-2 Design model for $2^{\text {nd }}$ case.

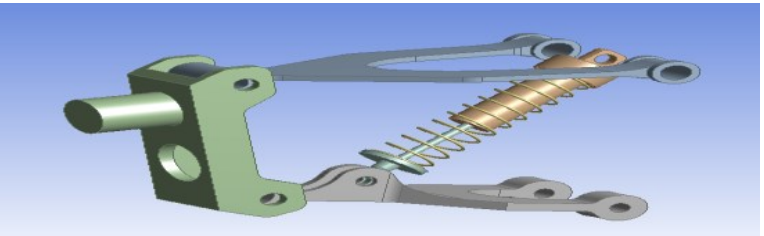

Figure 4: Single spring model prepared in CATIA

\section{CASE-3 Design model for $3^{\text {nd }}$ case.}

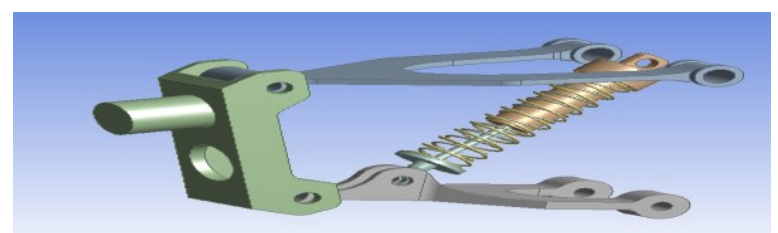

Figure 5: Double spring model prepared in CATIA

\section{Import Geometry in ANSYS for FEA Analysis}

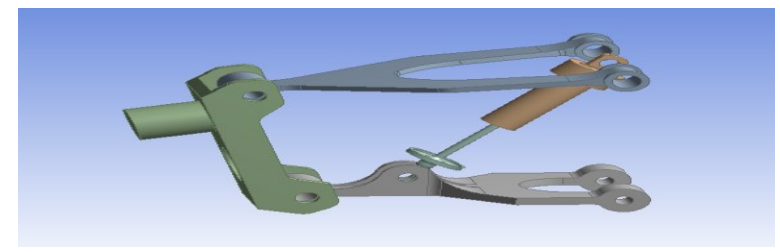

Figure 6: Import Geometry in ANSYS

\section{MESHING}

The mesh created in this work is shown in figure No 4.6. The total Node is generated 33685 \& Total No. of Elements is 31745 , it is clear from the mesh geometry the node numbers and element numbers are almost seven in digit which show that the mesh is very fine because the result accuracy depends on the mesh quality

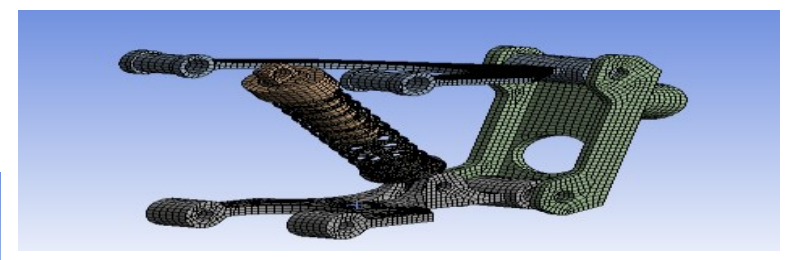

Figure 7: Meshing: Total No. of Nodes: 61915\& Total No. elements: 160624

\section{5) Boundary Condition}

1. Joints:-Number of joints are created according to pervious boundary condition.

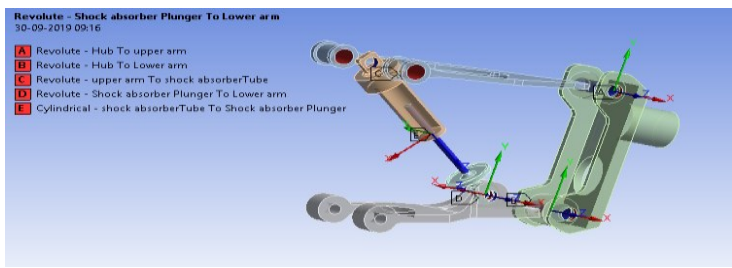

Figure 8: Define Joints

2. Spring element:-Define spring element between shock absorber plunger and shock absorber tube.

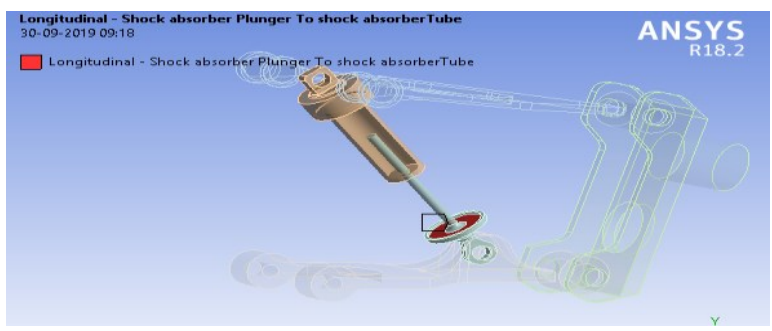

Figure 9: Define Spring element 


\section{Define Boundary conditions:-}

Forces were applied on the VSS in both radial and axial force per unit area with directions of about $14 \mathrm{kN} / \mathrm{m} 2$ and $2 \mathrm{kN} / \mathrm{m} 2$ respectively. The axial force was kept constant and the radial force was varied through 14, 16, 18, 20 and $22 \mathrm{kN} / \mathrm{m} 2$.

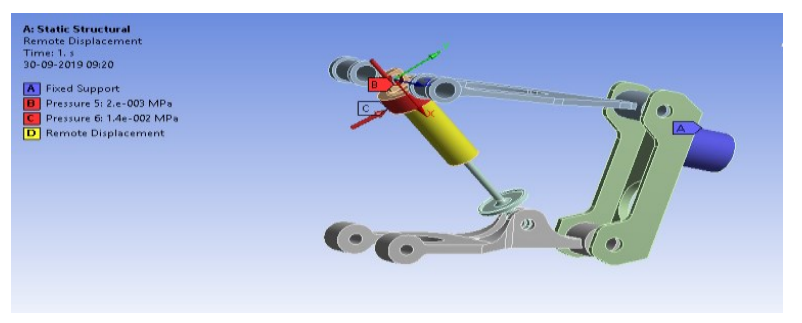

Figure 10: Define boundary conditions

4. Define fixed support:-Appling fixed support on VSS.

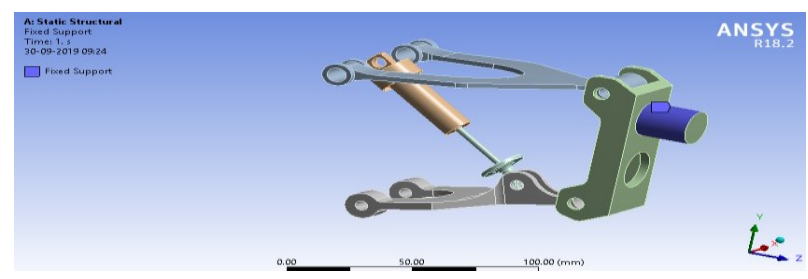

Figure 11: Fixed support

5. Define loading condition:-Applying axial force and radial force on vehicle suspension system.

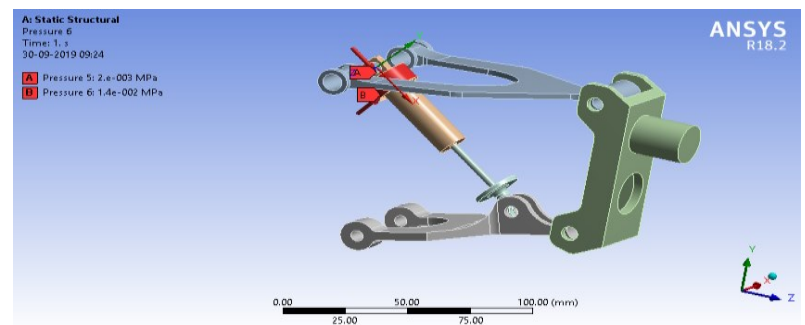

Figure 12: Loading condition

6. Define loading condition:-Control the six degree of freedom of absorber tube by applying Remote displacement.

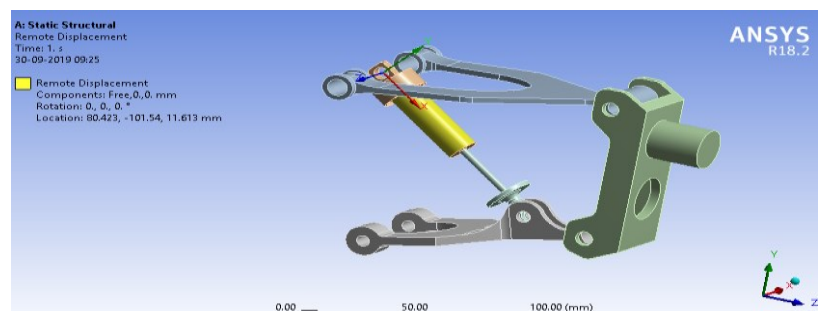

Figure 13: Displacement (Fixed translation in $\mathrm{Y}, \mathrm{Z}$ direction)

\section{6) Results OF $1^{\mathrm{ST}}$ Case}

The analysis of Cast iron at different load condition.

1. Equivalent stress:-In this case cast iron material is used for analysis at radial force $14 \mathbf{k N} / \mathbf{m}^{2}$

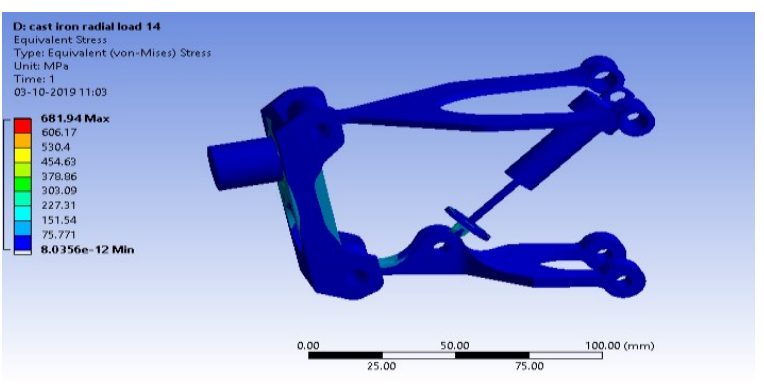

Figure 14: Equivalent stress at radial force $14 \mathrm{kN} / \mathrm{m}^{2}$

2. Equivalent stress:-Equivalent stress at radial force $16 \mathrm{kN} / \mathrm{m}^{2}$

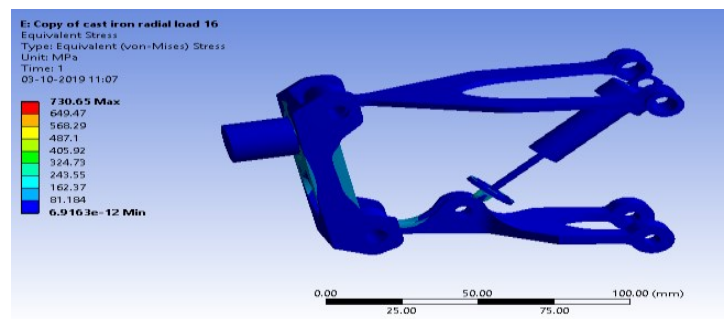

Figure 15: Equivalent stress at radial force $16 \mathrm{kN} / \mathrm{m}^{2}$

3. Equivalent stress:-Equivalent stress at radial force $18 \mathrm{kN} / \mathrm{m}^{2}$

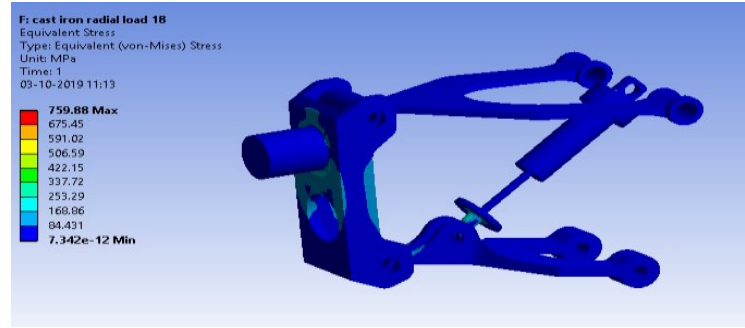

Figure 16: Equivalent stress at radial force $18 \mathrm{kN} / \mathrm{m}^{2}$

4. Equivalent stress:-Equivalent stress at radial force $20 \mathrm{kN} / \mathrm{m}^{2}$ 


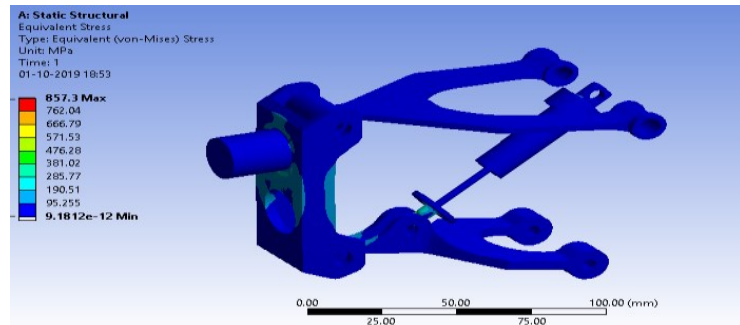

Figure 17: Equivalent stress at radial force $20 \mathrm{kN} / \mathrm{m}^{2}$

5. Equivalent stress:-Equivalent stress at radial force $22 \mathrm{kN} / \mathrm{m}^{2}$

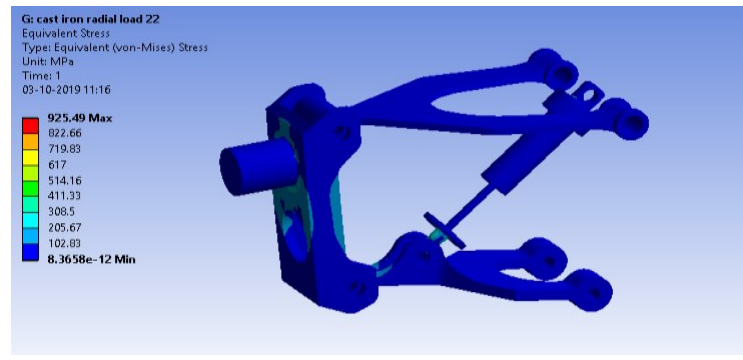

Figure 18: Equivalent stress at radial force $22 \mathrm{kN} / \mathrm{m}^{2}$

The analysis of Titanium Ti-13V-11Cr-3Al at different load condition.

1. Equivalent stress:-Case first material Titanium Ti$13 \mathrm{~V}-11 \mathrm{Cr}-3 \mathrm{Al}$ at radial force $14 \mathrm{kN} / \mathbf{m}^{2}$

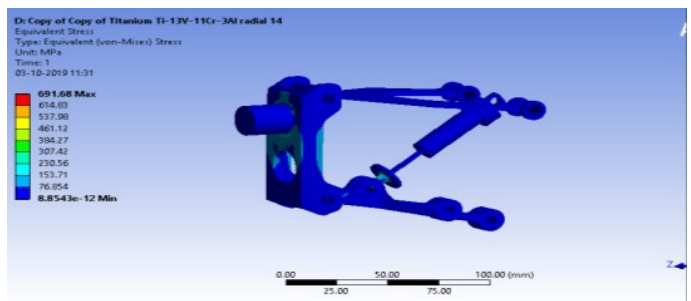

Figure 19: Equivalent stress at radial force $14 \mathrm{kN} / \mathrm{m}^{2}$

2. Equivalent stress:-Case first material Titanium Ti$13 \mathrm{~V}-11 \mathrm{Cr}-3 \mathrm{Al}$ at radial force $\mathbf{1 6} \mathbf{k N} / \mathbf{m}^{2}$

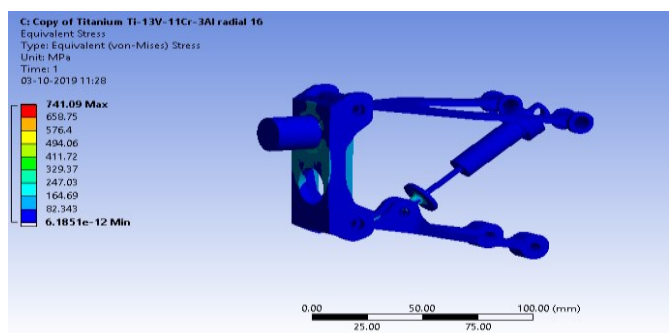

Figure 20: Equivalent stress at radial force $16 \mathrm{kN} / \mathrm{m}^{2}$

3. Equivalent stress:-Case first material Titanium Ti$13 \mathrm{~V}-11 \mathrm{Cr}-3 \mathrm{Al}$ at radial force $18 \mathrm{kN} / \mathbf{m}^{2}$

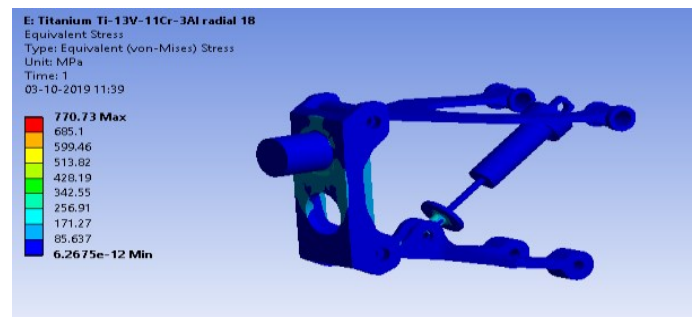

Figure 21: Equivalent stress at radial force $18 \mathrm{kN} / \mathrm{m}^{2}$

4. Equivalent stress:-Case first material Titanium Ti$13 \mathrm{~V}-11 \mathrm{Cr}-3 \mathrm{Al}$ at radial force $20 \mathrm{kN} / \mathbf{m}^{2}$

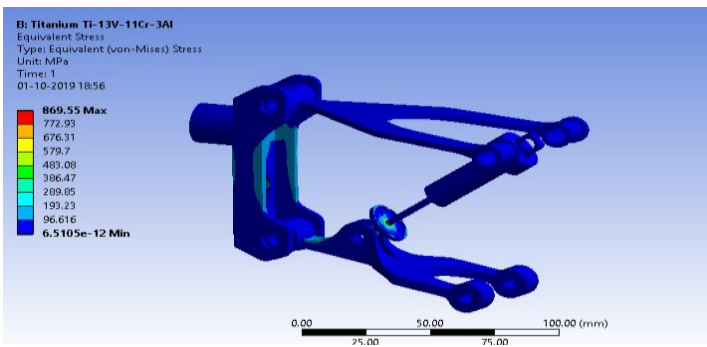

Figure 22: Equivalent stress at radial force $20 \mathrm{kN} / \mathrm{m}^{2}$

5. Equivalent stress:-Case first material Titanium Ti$13 \mathrm{~V}-11 \mathrm{Cr}-3 \mathrm{Al}$ at radial force $22 \mathrm{kN} / \mathbf{m}^{2}$

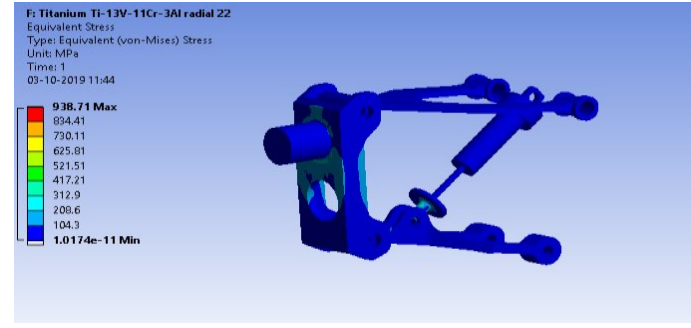

Figure 23: Equivalent stress at radial force $20 \mathrm{kN} / \mathrm{m}^{2}$

The analysis of Titanium Ti-6Al-4Vat different load condition.

1. Equivalent stress:-Case first material Titanium Ti$6 \mathrm{Al}-4 \mathrm{Vat}$ radial force $14 \mathbf{k N} / \mathbf{m}^{2}$

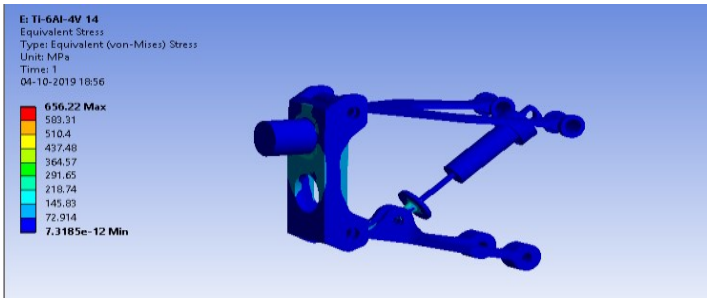

Figure 24: Equivalent stress at radial force $14 \mathrm{kN} / \mathrm{m}^{2}$

2. Equivalent stress:-Case first material Titanium Ti$6 \mathrm{Al}-4 \mathrm{~V}$ at radial force $16 \mathrm{kN} / \mathbf{m}^{2}$ 


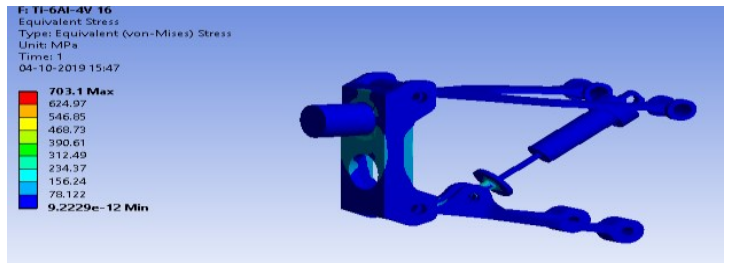

Figure 25: Equivalent stress at radial force $16 \mathrm{kN} / \mathrm{m}^{2}$

3. Equivalent stress:-Case first material Titanium Ti$6 \mathrm{Al}-4 \mathrm{~V}$ at radial force $18 \mathrm{kN} / \mathbf{m}^{2}$

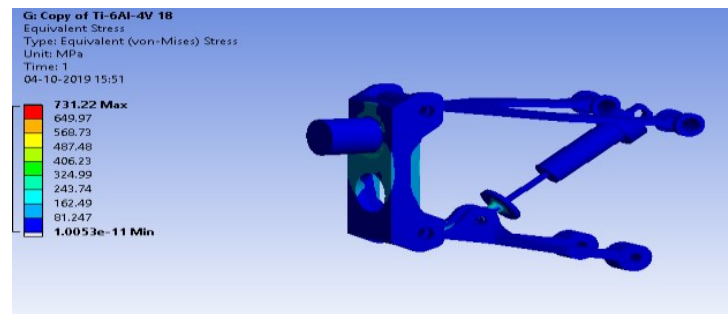

Figure 26: Equivalent stress at radial force $18 \mathrm{kN} / \mathrm{m}^{2}$

4. Equivalent stress:-Case first material Titanium Ti$6 \mathrm{Al}-4 \mathrm{~V}$ at radial force $20 \mathrm{kN} / \mathbf{m}^{2}$

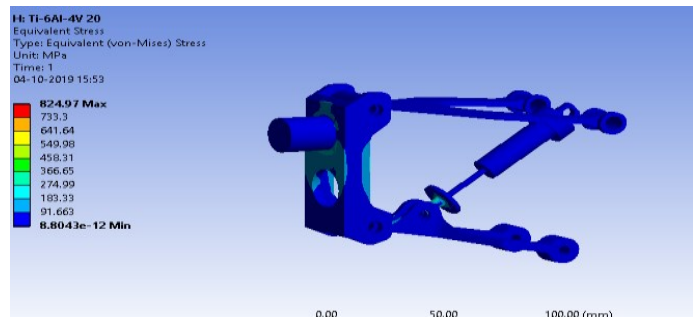

Figure 27: Equivalent stress at radial force $20 \mathrm{kN} / \mathrm{m}^{2}$

5. Equivalent stress:-Case first material Titanium Ti$6 \mathrm{Al}-4 \mathrm{Vat}$ radial force $22 \mathrm{kN} / \mathbf{m}^{2}$

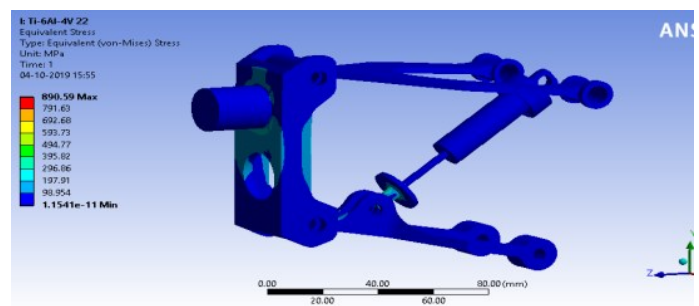

Figure 28: Equivalent stress at radial force $20 \mathrm{kN} / \mathrm{m}^{2}$

The analysis of Aluminum 7075-T6 at different load condition.

1. Equivalent stress:-Case first material at radial force $14 \mathrm{kN} / \mathrm{m}^{2}$

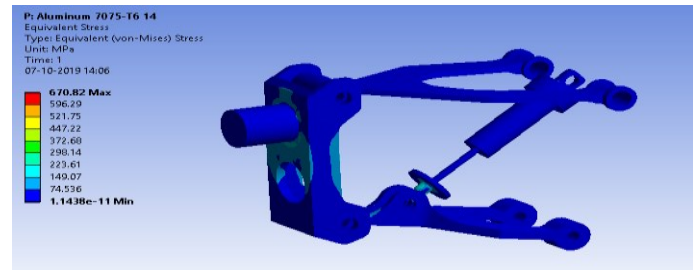

Figure 29: Equivalent stress at radial force $14 \mathrm{kN} / \mathrm{m}^{2}$

2. Equivalent stress:-Case first material Aluminum 7075-T6at radial force $16 \mathbf{k N} / \mathbf{m}^{2}$

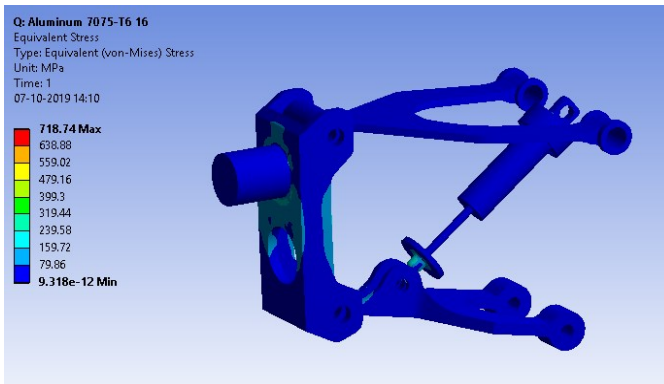

Figure 30: Equivalent stress at radial force $16 \mathrm{kN} / \mathrm{m}^{2}$

3. Equivalent stress:-Case first material Aluminum 7075-T6at radial force $\mathbf{1 8 k N} / \mathbf{m}^{2}$

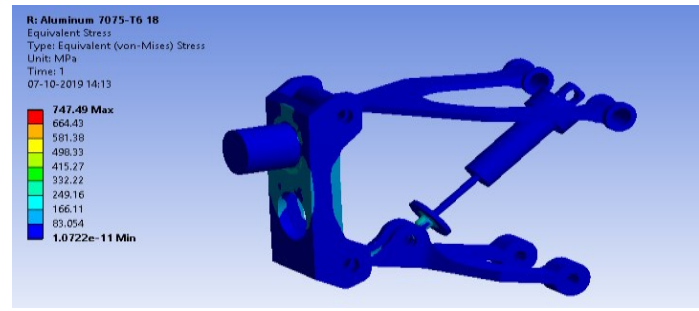

Figure 31: Equivalent stress at radial force $18 \mathrm{kN} / \mathrm{m}^{2}$

4. Equivalent stress:-Case first material Aluminum 7075-T6 at radial force $20 \mathbf{k N} / \mathbf{m}^{2}$

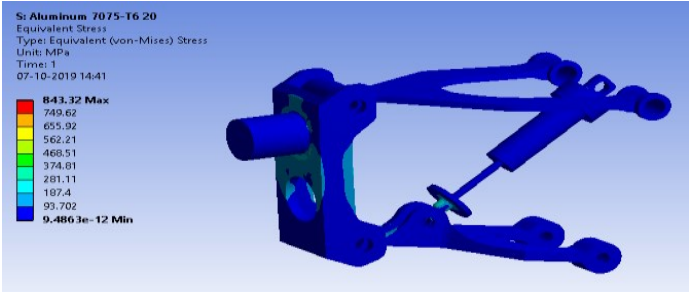

Figure 32: Equivalent stress at radial force $20 \mathrm{kN} / \mathrm{m}^{2}$

5. Equivalent stress:-Case first material Aluminum 7075-T6 at radial force $22 \mathrm{kN} / \mathbf{m}^{2}$ 


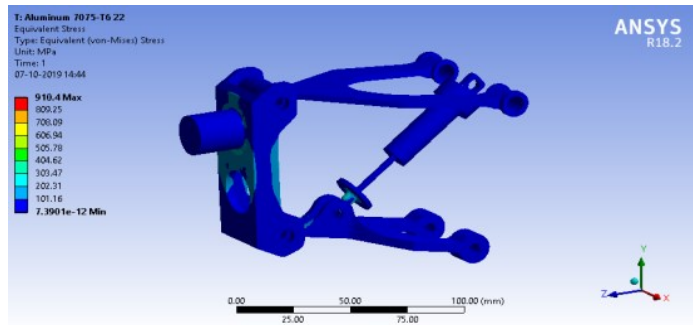

Figure 33: Equivalent stress at radial force $22 \mathrm{kN} / \mathrm{m}^{2}$

\section{7) Results of $2^{\mathrm{ST}}$ Case}

1. Equivalent stress:-Case first material cast iron at radial force $14 \mathrm{kN} / \mathrm{m}^{2}$

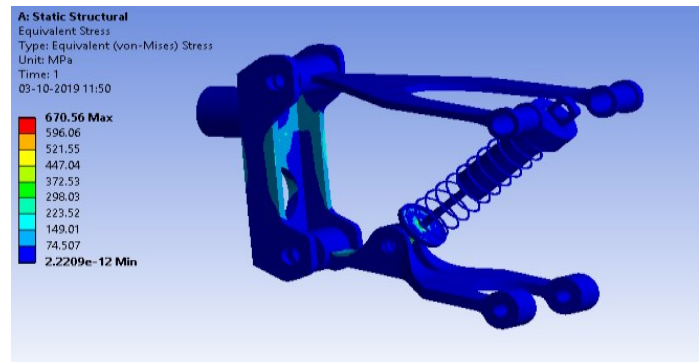

Figure 34: Equivalent stress at radial force $14 \mathrm{kN} / \mathrm{m}^{2}$

2. Equivalent stress:-Case first material cast iron at radial force $16 \mathrm{kN} / \mathrm{m}^{2}$

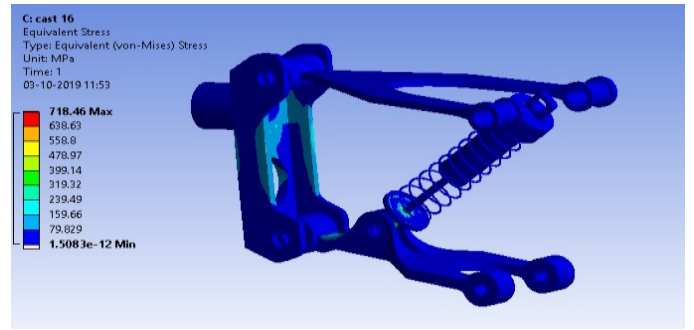

Figure 35: Equivalent stress at radial force $16 \mathrm{kN} / \mathrm{m}^{2}$

3. Equivalent stress:-Case first material cast iron at radial force $18 \mathrm{kN} / \mathbf{m}^{2}$

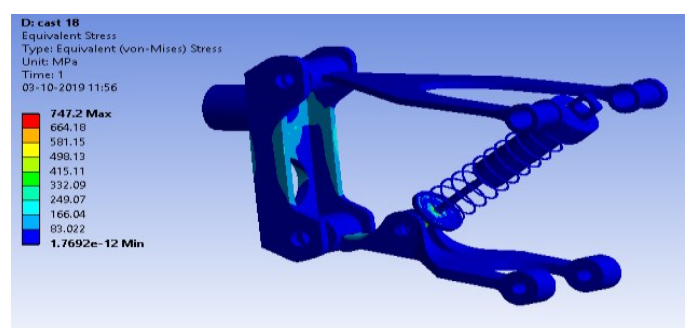

Figure 36: Equivalent stress at radial force $18 \mathrm{kN} / \mathrm{m}^{2}$

4. Equivalent stress:-Case first material cast iron at radial force $20 \mathrm{kN} / \mathbf{m}^{2}$

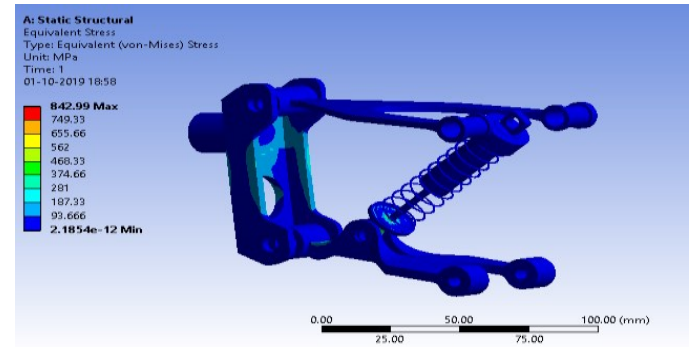

Figure 37: Equivalent stress at radial force $20 \mathrm{kN} / \mathrm{m}^{2}$

5. Equivalent stress:-Case first material cast iron at radial force $22 \mathrm{kN} / \mathbf{m}^{2}$

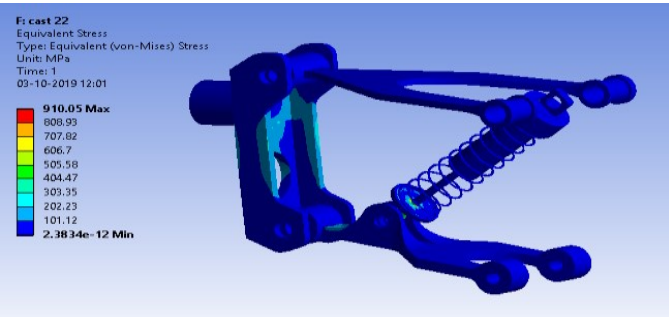

Figure 38: Equivalent stress at radial force $22 \mathrm{kN} / \mathrm{m}^{2}$

8) The analysis is to be done on the consideration of single spring in Titanium Ti-13V-11Cr-3Al at different loading conditions.

1. Equivalent stress:-Case first material Titanium Ti$13 \mathrm{~V}-11 \mathrm{Cr}-3 \mathrm{Al}$ at radial force $\mathbf{1 4} \mathbf{k N} / \mathbf{m}^{2}$

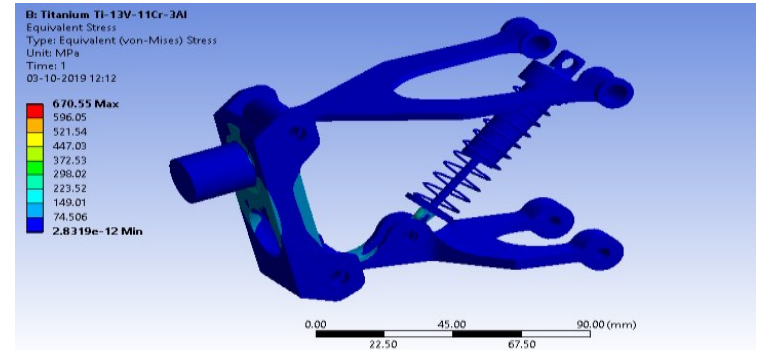

Figure 39: Equivalent stress at radial force $14 \mathrm{kN} / \mathrm{m}^{2}$

2. Equivalent stress:-Case first material Titanium Ti$13 \mathrm{~V}-11 \mathrm{Cr}-3 \mathrm{Al}$ at radial force $\mathbf{1 6} \mathbf{k N} / \mathbf{m}^{2}$

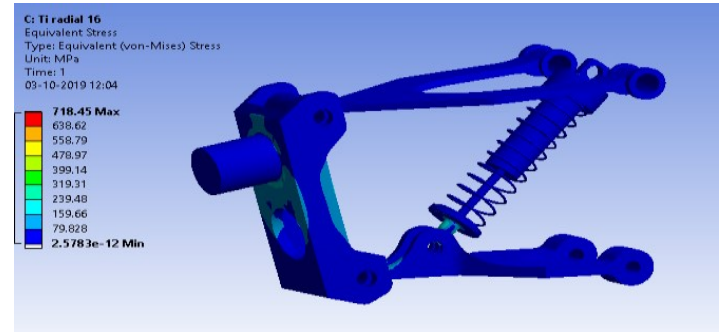

Figure 40: Equivalent stress at radial force $16 \mathrm{kN} / \mathrm{m}^{2}$ 
3. Equivalent stress:-Case first material Titanium Ti$13 \mathrm{~V}-11 \mathrm{Cr}-3 \mathrm{Al}$ at radial force $\mathbf{1 8} \mathbf{k N} / \mathbf{m}^{2}$

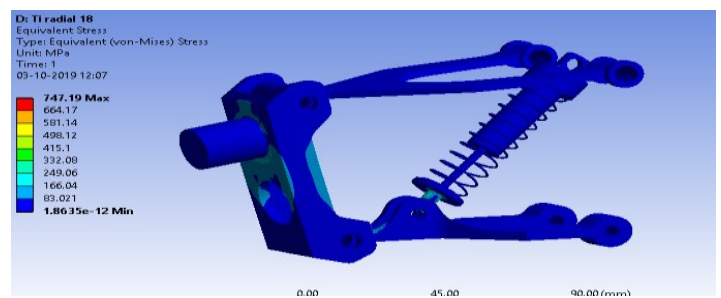

Figure 41.: Equivalent stress at radial force $18 \mathrm{kN} / \mathrm{m}^{2}$

4. Equivalent stress:-Case first material Titanium Ti$13 \mathrm{~V}-11 \mathrm{Cr}-3 \mathrm{Al}$ at radial force $20 \mathrm{kN} / \mathbf{m}^{2}$

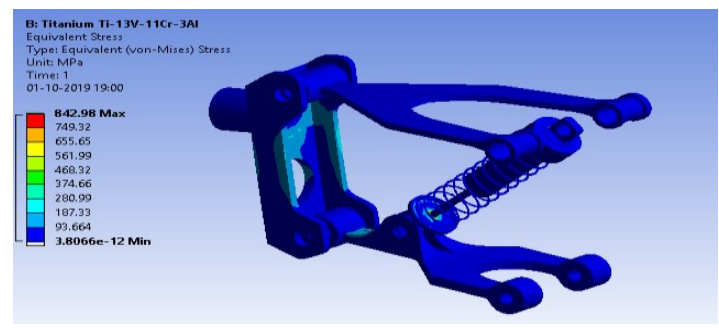

Figure 42: Equivalent stress at radial force $20 \mathrm{kN} / \mathrm{m}^{2}$

5. Equivalent stress:-Case first material Titanium $\mathrm{Ti}$ $13 \mathrm{~V}-11 \mathrm{Cr}-3 \mathrm{Al}$ at radial force $22 \mathrm{kN} / \mathbf{m}^{2}$

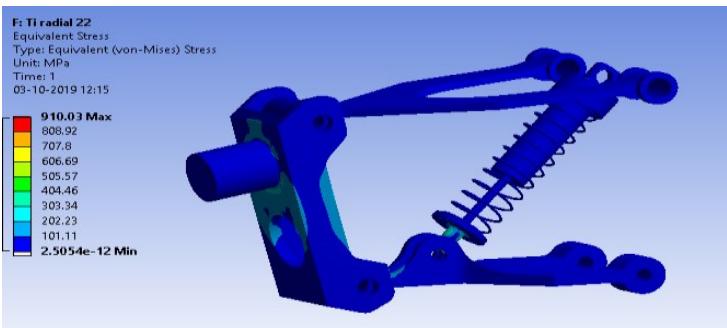

Figure 43: Equivalent stress at radial force $22 \mathrm{kN} / \mathrm{m}^{2}$

9) The analysis is to be done on the consideration of single spring in Titanium Ti-6Al-4V at different loading conditions.

1. Equivalent stress:-Case first material Titanium Ti$6 \mathrm{Al}-4$ Vat radial force $\mathbf{1 4} \mathbf{k N} / \mathbf{m}^{2}$

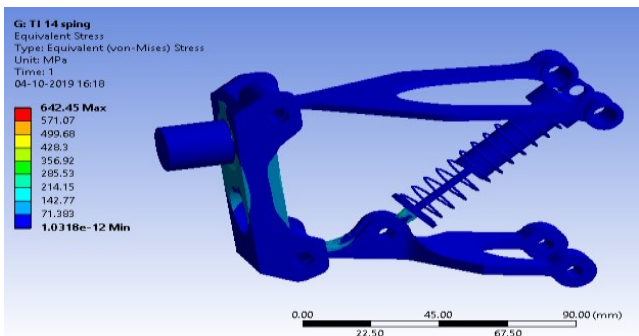

Figure 44: Equivalent stress at radial force $14 \mathrm{kN} / \mathrm{m}^{2}$
2. Equivalent stress:-Case first material Titanium Ti$6 \mathrm{Al}-4$ Vat radial force $\mathbf{1 6} \mathrm{kN} / \mathbf{m}^{2}$

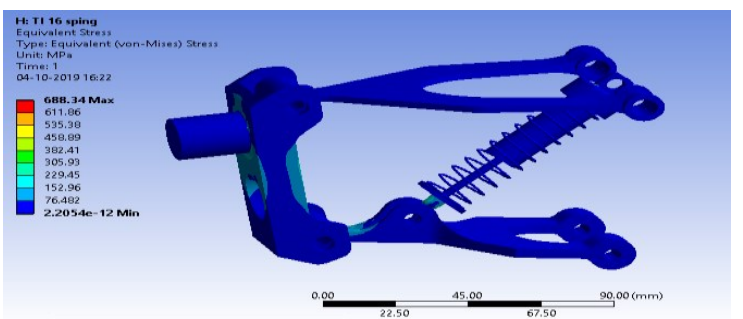

Figure 45: Equivalent stress at radial force $16 \mathrm{kN} / \mathrm{m}^{2}$

3. Equivalent stress:-Case first material Titanium Ti$6 \mathrm{Al}-4$ Vat radial force $\mathbf{1 8 k N} / \mathbf{m}^{2}$

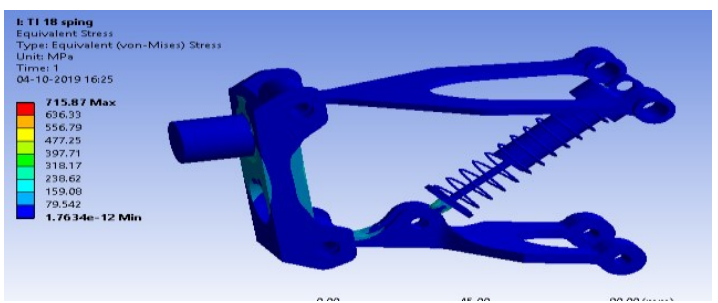

Figure 46: Equivalent stress at radial force $18 \mathrm{kN} / \mathrm{m}^{2}$

4. Equivalent stress:-Case first material Titanium Ti$6 \mathrm{Al}-4$ Vat radial force $20 \mathrm{kN} / \mathbf{m}^{2}$

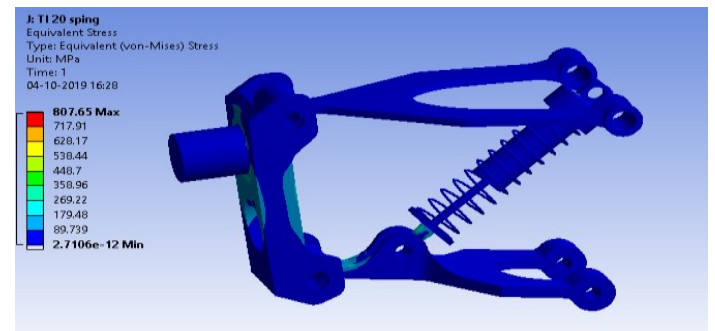

Figure 47: Equivalent stress at radial force $20 \mathrm{kN} / \mathrm{m}^{2}$

5. Equivalent stress:-Case first material Titanium Ti$6 \mathrm{Al}-4$ Vat radial force $22 \mathrm{kN} / \mathbf{m}^{2}$

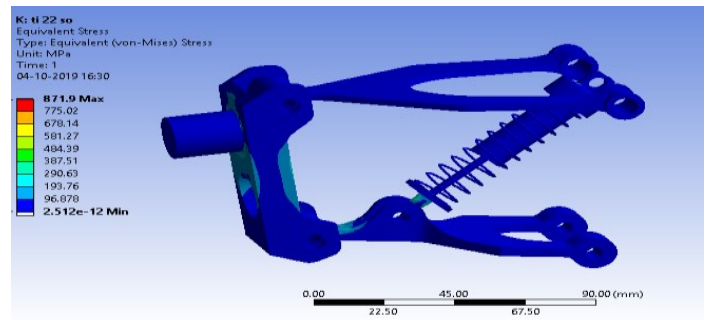

Figure 48: Equivalent stress at radial force $22 \mathrm{kN} / \mathrm{m}^{2}$

10) The analysis is to be done on the consideration of single spring in Aluminum 7075-T6 at different loading conditions. 
1. Equivalent stress:- Case second material Aluminum 7075-T6 at radial force $\mathbf{1 4 k N} / \mathbf{m}^{2}$

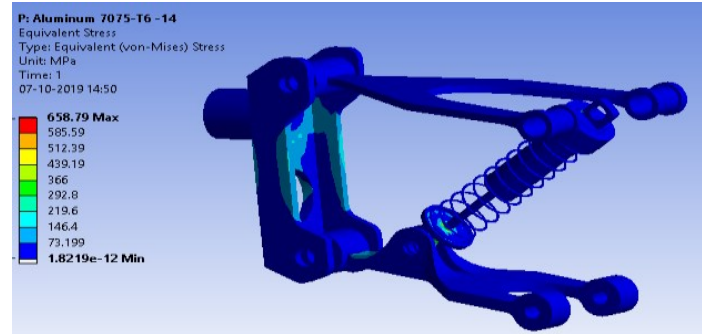

Figure 49: Equivalent stress at radial force $14 \mathrm{kN} / \mathrm{m}^{2}$

2. Equivalent stress:-Case second material Aluminum $7075-\mathrm{T} 6$ at radial force $16 \mathrm{kN} / \mathrm{m}^{2}$

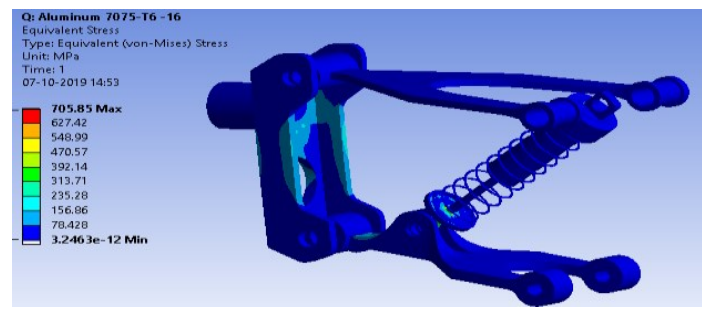

Figure 50: Equivalent stress at radial force $16 \mathrm{kN} / \mathrm{m}^{2}$

3. Equivalent stress:-Case second material Aluminum $7075-\mathrm{T} 6$ at radial force $18 \mathrm{kN} / \mathrm{m}^{2}$

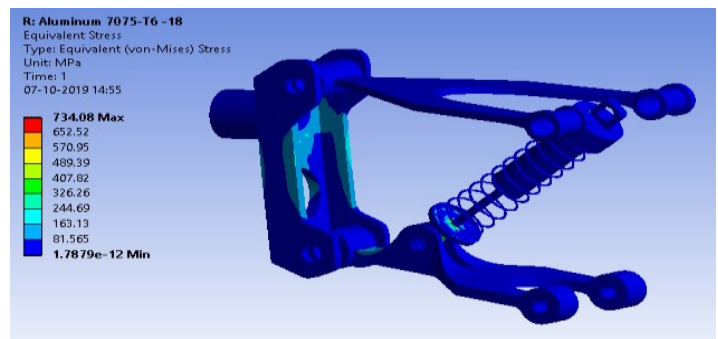

Figure 51: Equivalent stress at radial force $18 \mathrm{kN} / \mathrm{m}^{2}$

4. Equivalent stress:-Case second material Aluminum 7075-T6 at radial force $20 \mathrm{kN} / \mathrm{m}^{2}$

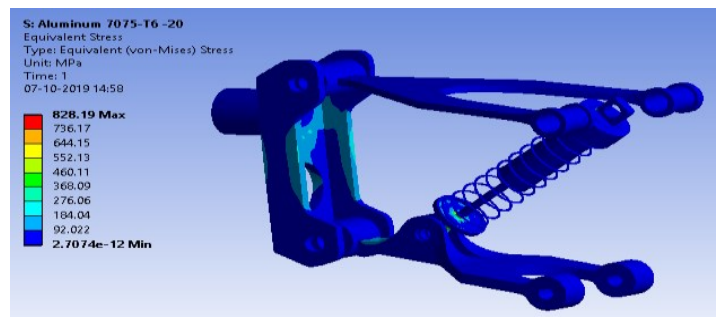

Figure 52: Equivalent stress at radial force $20 \mathrm{kN} / \mathrm{m}^{2}$

5. Equivalent stress:-Case second material Aluminum $7075-\mathrm{T} 6$ at radial force $22 \mathrm{kN} / \mathrm{m}^{2}$

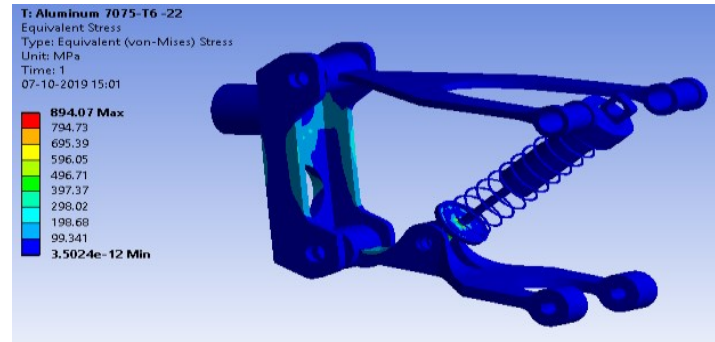

Figure 53: Equivalent stress at radial force $22 \mathrm{kN} / \mathrm{m}^{2}$

\section{1) Results OF $3^{\text {rd }}$ Case}

Adding spring in this case, and the analysis is to be done on the consideration of double spring in cast iron at different loading conditions.

1. Equivalent stress:- Case first material Cast Iron at radial force $14 \mathrm{kN} / \mathbf{m}^{2}$

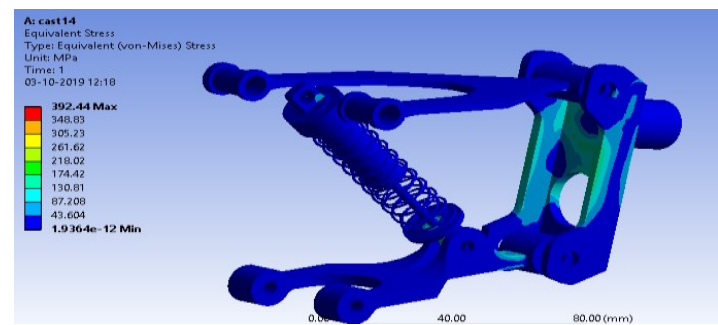

Figure 54: Equivalent stress at radial force $14 \mathrm{kN} / \mathrm{m}^{2}$

2. Equivalent stress:-Case first material Cast Iron at radial force $16 \mathrm{kN} / \mathbf{m}^{2}$

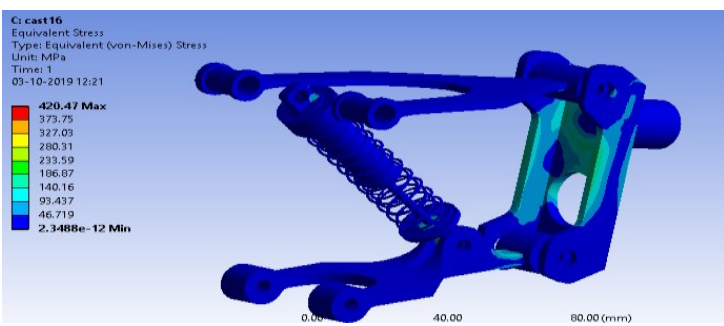

Figure 55: Equivalent stress at radial force $16 \mathrm{kN} / \mathrm{m}^{2}$

3. Equivalent stress:-Case first material Cast Iron at radial force $20 \mathrm{kN} / \mathbf{m}^{2}$

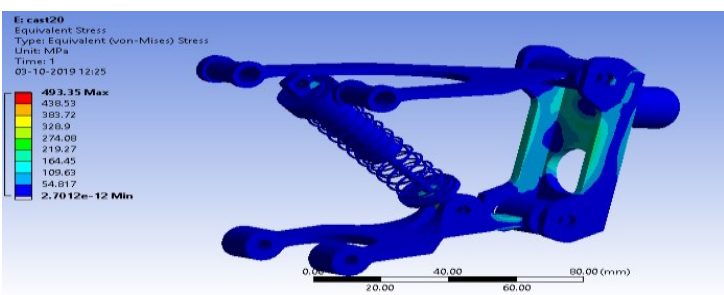

Figure 56: Equivalent stress at radial force $20 \mathrm{kN} / \mathrm{m}^{2}$ 
4. Equivalent stress:-Case first material Cast Iron at radial force $22 \mathrm{kN} / \mathbf{m}^{2}$

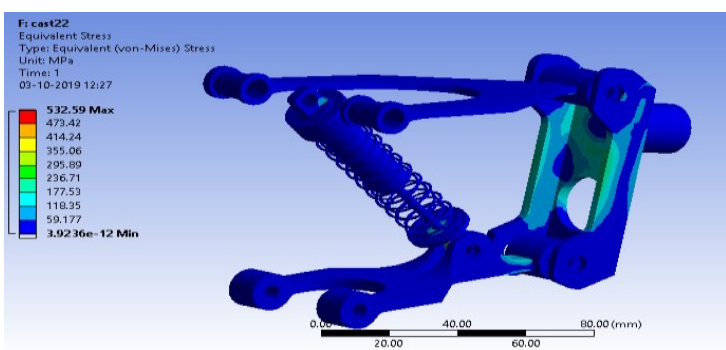

Figure 57: Equivalent stress at radial force $22 \mathrm{kN} / \mathrm{m}^{2}$

12) The analysis is to be done on the consideration of double spring in Titanium Ti-13V-11Cr-3Al at different loading conditions.

1. Equivalent stress:-Case first material Titanium Ti$13 \mathrm{~V}-11 \mathrm{Cr}-3 \mathrm{Al}$ at radial force $\mathbf{1 4} \mathbf{~ k N} / \mathbf{m}^{2}$

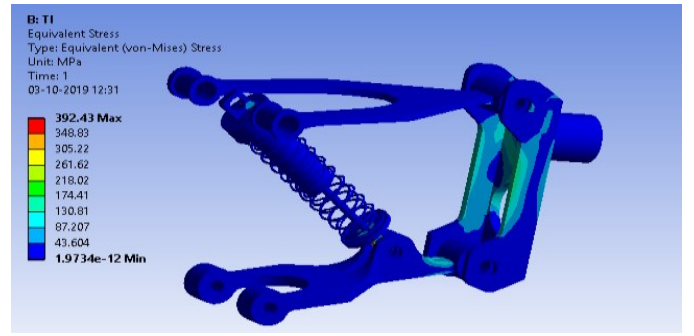

Figure 58: Equivalent stress at radial force $14 \mathrm{kN} / \mathrm{m}^{2}$

2. Equivalent stress:-Case first material Titanium $\mathrm{Ti}$ $13 \mathrm{~V}-11 \mathrm{Cr}-3 \mathrm{Al}$ at radial force $\mathbf{1 6} \mathbf{~ k N} / \mathbf{m}^{2}$

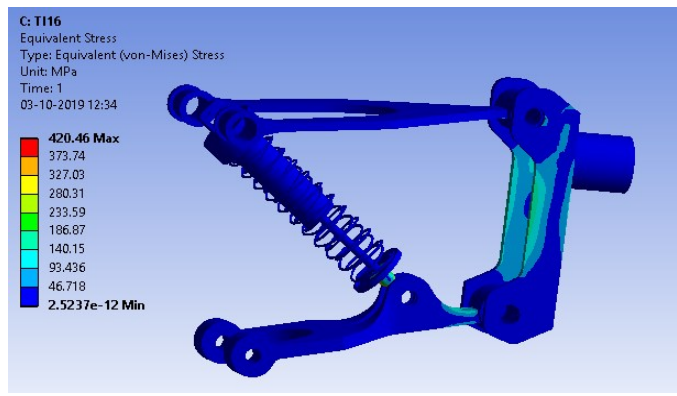

Figure 59: Equivalent stress at radial force $16 \mathrm{kN} / \mathrm{m}^{2}$

3. Equivalent stress:-Case first material Titanium Ti$13 \mathrm{~V}-11 \mathrm{Cr}-3 \mathrm{Al}$ at radial force $\mathbf{1 8} \mathbf{k N} / \mathbf{m}^{2}$

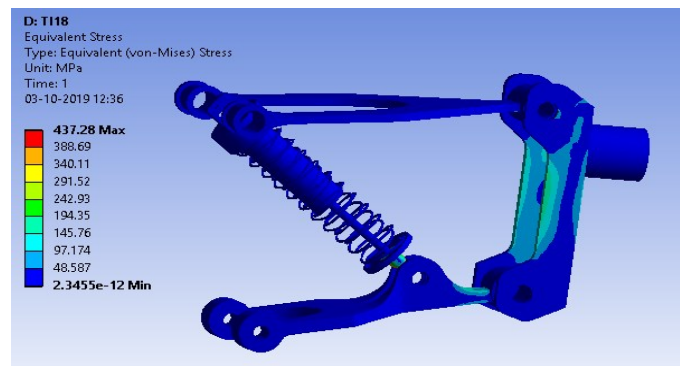

Figure 60: Equivalent stress at radial force $18 \mathrm{kN} / \mathrm{m}^{2}$

4. Equivalent stress:-Case first material Titanium Ti$13 \mathrm{~V}-11 \mathrm{Cr}-3 \mathrm{Al}$ at radial force $20 \mathrm{kN} / \mathbf{m}^{2}$

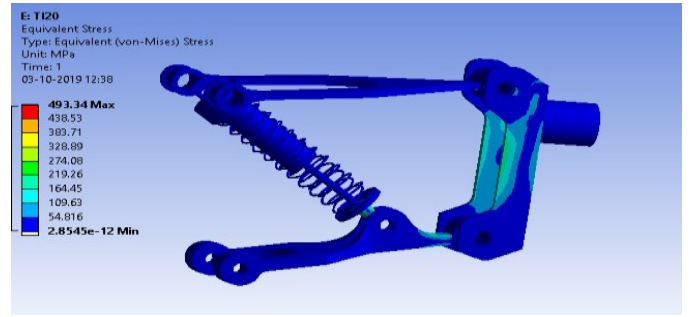

Figure 61: Equivalent stress at radial force $20 \mathrm{kN} / \mathrm{m}^{2}$

13) The analysis is to be done on the consideration of double spring Titanium Ti-6Al-4V at different loading conditions.

1. Equivalent stress:-Case first material Titanium Ti6Al-4Vat radial force $14 \mathrm{kN} / \mathbf{m}^{2}$

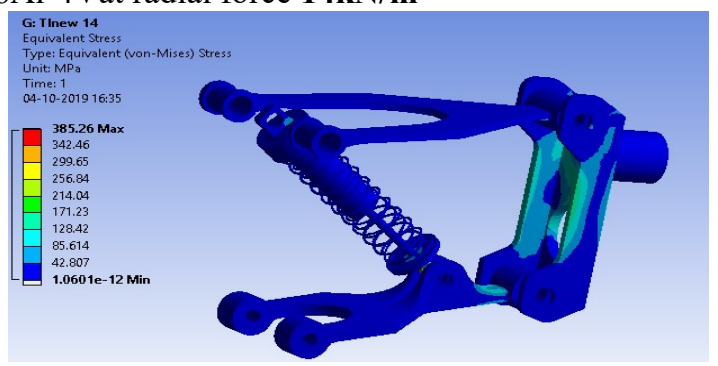

Figure 62: Equivalent stress at radial force $14 \mathrm{kN} / \mathrm{m}^{2}$

3. Equivalent stress:-Case first material Titanium Ti6Al-4Vat radial force $16 \mathrm{kN} / \mathbf{m}^{2}$

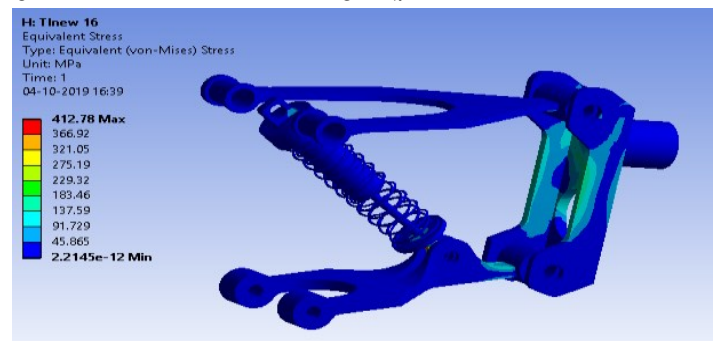

Figure 63: Equivalent stress at radial force $16 \mathrm{kN} / \mathrm{m}^{2}$

4. Equivalent stress:-Case first material Titanium Ti$6 \mathrm{Al}-4$ Vat radial force $\mathbf{1 8 k N} / \mathbf{m}^{2}$ 


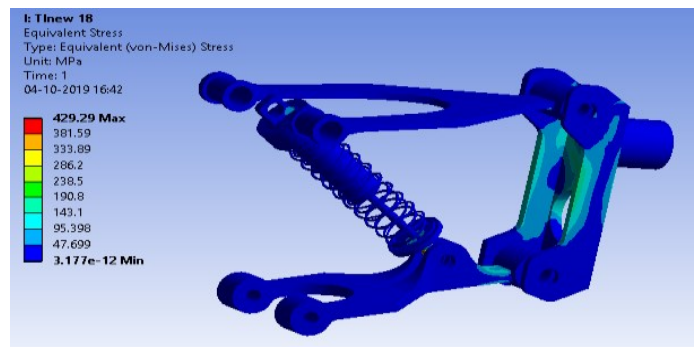

Figure 64: Equivalent stress at radial force $18 \mathrm{kN} / \mathrm{m}^{2}$

5. Equivalent stress:-Case first material Titanium Ti$6 \mathrm{Al}-4 \mathrm{Vat}$ radial force $20 \mathrm{kN} / \mathbf{m}^{2}$

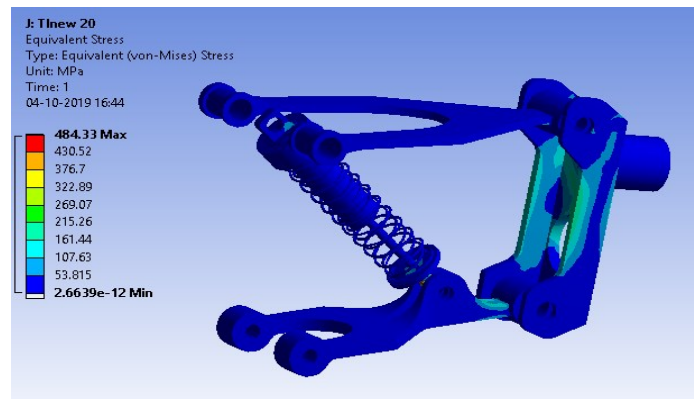

Figure 65: Equivalent stress at radial force $20 \mathrm{kN} / \mathrm{m}^{2}$

6. Equivalent stress:-Case first material Titanium Ti$6 \mathrm{Al}-4 \mathrm{~V}$ at radial force $22 \mathrm{kN} / \mathbf{m}^{2}$

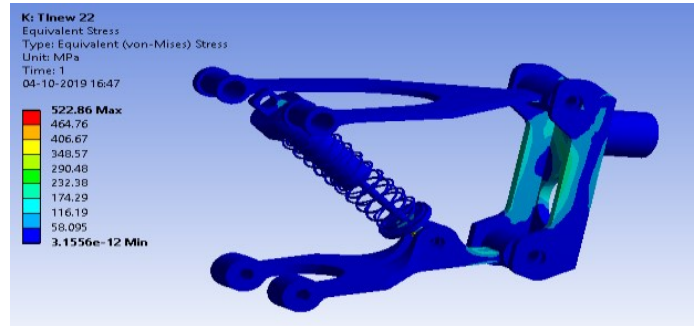

Figure 66: Equivalent stress at radial force $22 \mathrm{kN} / \mathrm{m}^{2}$

14) In this case adding springs with proposed material Aluminum 7075-T6 in the vehicle suspension system.

1. Equivalent stress:-Case third material Aluminum $7075-\mathrm{T} 6$ at radial force $14 \mathbf{k N} / \mathbf{m}^{2}$

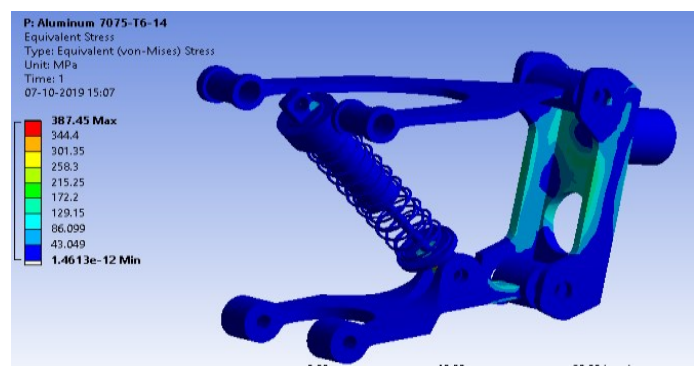

Figure 67: Equivalent stress at radial force $14 \mathrm{kN} / \mathrm{m}^{2}$
2. Equivalent stress:-Case third material Aluminum 7075-T6 at radial force $16 \mathbf{k N} / \mathbf{m}^{2}$

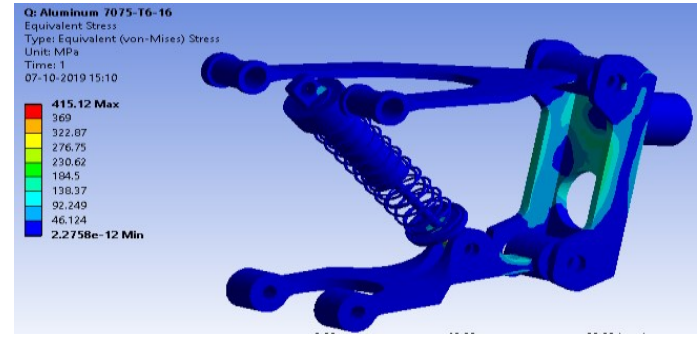

Figure 68: Equivalent stress at radial force $16 \mathrm{kN} / \mathrm{m}^{2}$

3. Equivalent stress:-Case third material Aluminum 7075-T6 at radial force $18 \mathrm{kN} / \mathbf{m}^{\mathbf{2}}$

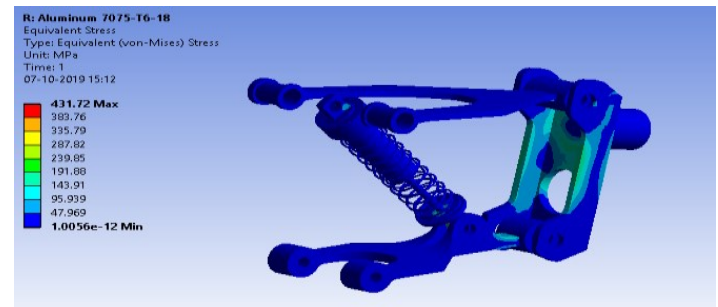

Figure 69: Equivalent stress at radial force $18 \mathrm{kN} / \mathrm{m}^{2}$

4. Equivalent stress:-Case third material Aluminum 7075-T6 at radial force $20 \mathrm{kN} / \mathbf{m}^{2}$

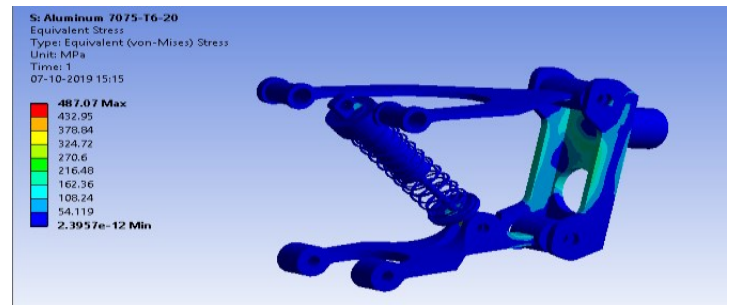

Figure 70: Equivalent stress at radial force $20 \mathrm{kN} / \mathrm{m}^{2}$

5. Equivalent stress:-Case third material Aluminum 7075-T6 at radial force $22 \mathrm{kN} / \mathbf{m}^{2}$

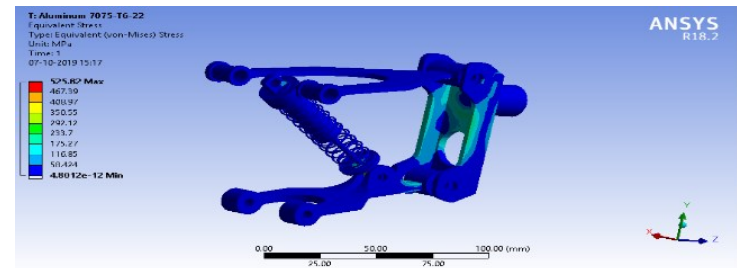

Figure 71: Equivalent stress at radial force $22 \mathrm{kN} / \mathrm{m}^{2}$

\section{RESULTS}

1) FEA ANALYSIS ON VEHICLE SUSPENSION SYSTEM 


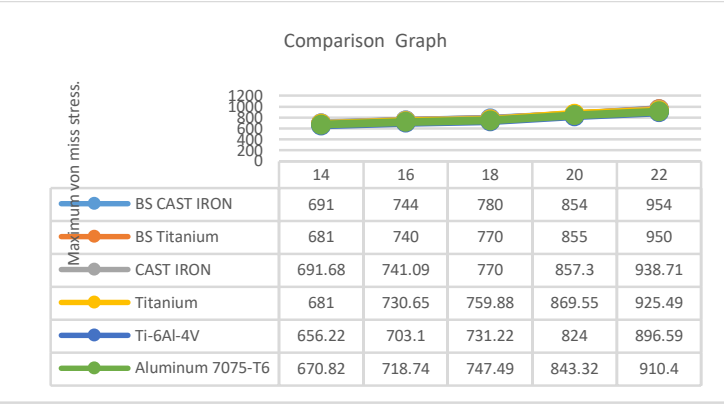

Graph 1 Maximum von miss stress of the materials.

2) Cast iron result in case 1,2 , and 3 .

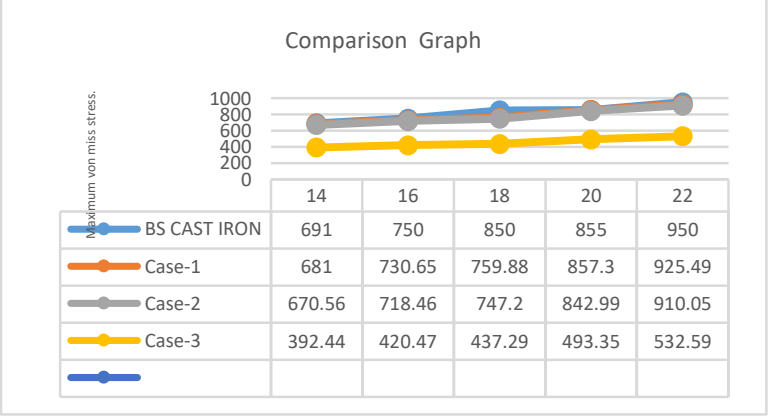

Graph 2 Maximum von miss stress of the cast iron material.

3) Titanium result in case 1,2 , and 3 .

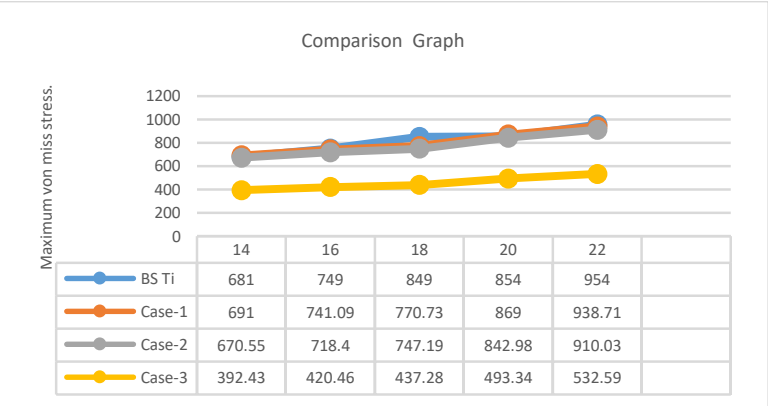

Graph 3 Maximum von miss stress of the Titanium materials.

4) Titanium Ti-6Al-4V result in case 1,2 , and 3.

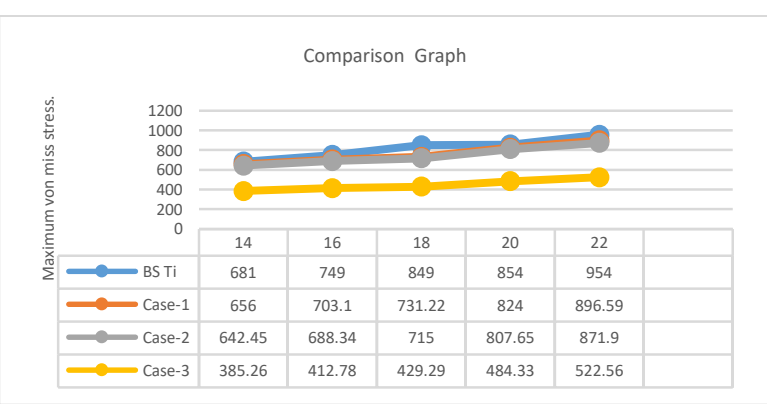

Graph 4 Maximum von miss stress of the Titanium Ti6Al-4V materials.

5) Aluminum 7075-T6 result in case 1, 2, and 3.

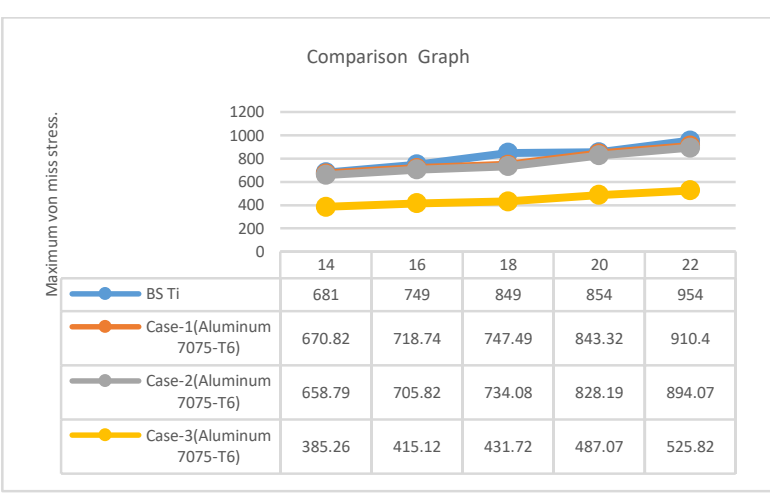

Graph 5 Maximum von miss stress of the Aluminum 7075-T6 materials.

6) Factor of safety of VSS (Titanium result in case 1, 2, and 3.)

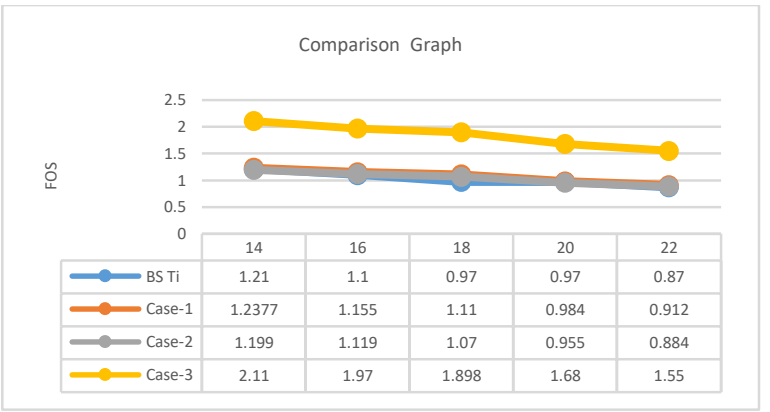

Graph 6 Comparison Graph Titanium (Factor of safety of VSS)

7) Factor of safety of VSS (Cast Iron result in case 1, 2, and 3.)

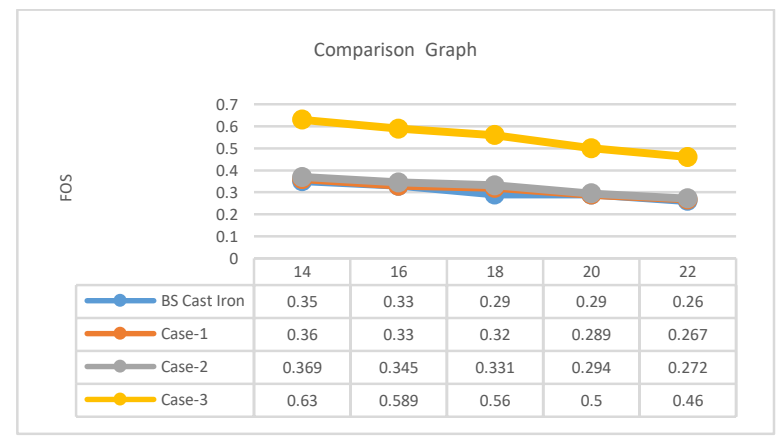

Graph 7 Comparison Graph Cast Iron (Factor of safety of VSS)

8) Factor of safety of VSS (Titanium Ti-6Al-4V result in case 1, 2, and 3.) 


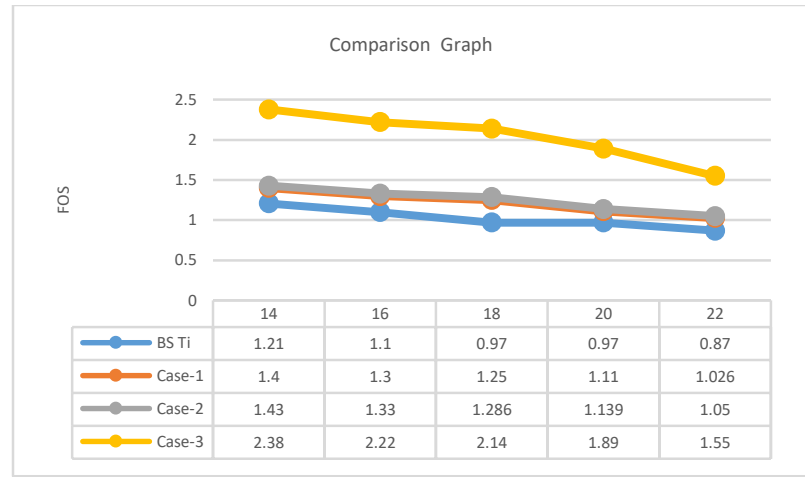

Graph 8 Comparison Graph Titanium Ti-6Al-4V (Factor of safety of VSS)

9) Factor of safety of VSS (Aluminum 7075-T6 result in case 1, 2, and 3.)

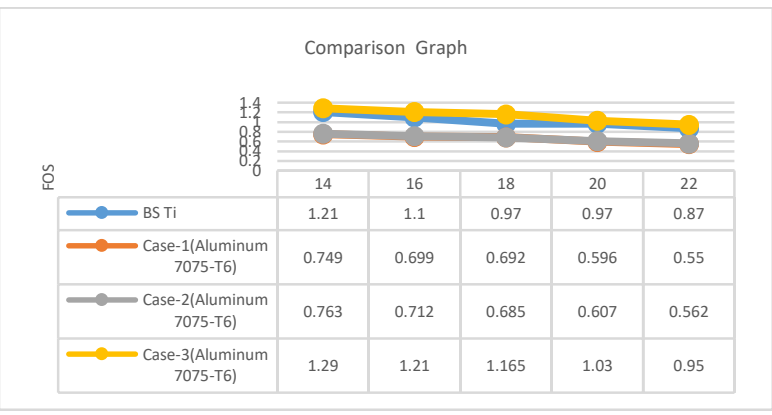

Graph 9 Comparison Graph Aluminum 7075-T6 (Factor of safety of VSS)

\section{0) Weight Comparison Graph.}

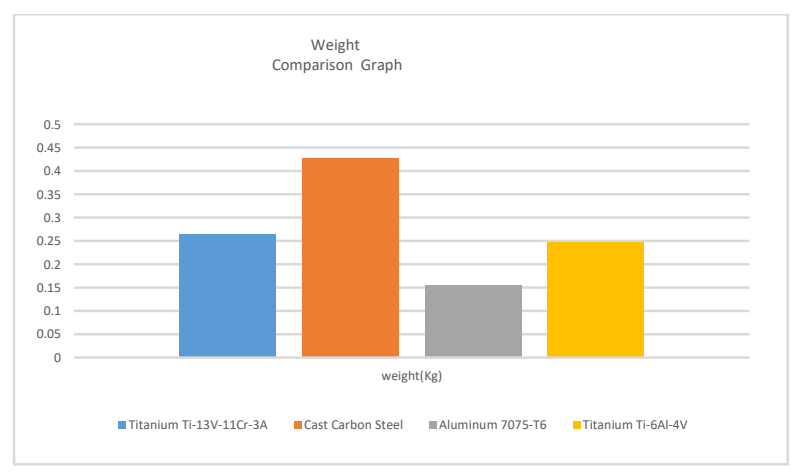

Graph 10 Weight Comparison Graph

\section{CONCLUSION}

The analysis of the duration and static stresses of a VSS using finite element analysis techniques has provided a reliable design that can be used in VSS projects. This work presents a fatigue index for the SSV and based on the simulation obtained, we can say that:
- The current VSS made of a carbon alloy can be reduced to a lighter VSS with good durability and good workability with the advantage of low $\mathrm{CO} 2$ emissions.

- The FOS for carbon alloys with an adequate elasticity point lower than the VMS value is about 0.50 for the different loads considered.

- Titanium Ti-6Al-4VSolution Treated has an FOS of approximately 2.38 in $3^{\text {rd }}$ case. The total strength of the Titanium is increase due to double spring. The value of equilavant stress decrease in case $3^{\text {rd }}$.and total factor of safety increases up to 2.38 in third case.

- The value of maximum von mises stress decrease in case third and the value of FOS increases. The value of Factor of safety in case third maximum 2.38 and minimum 1.76 .

- But in Case of cast iron the value of FOS 0.63 at $14 \mathrm{kN} / \mathrm{m} 3$ radial force. And 0.46 at $22 \mathrm{kN} / \mathrm{m} 2$.

- The total weight of Aluminum 7075-T6 is lower than the other material. And factor of safety of the aluminum in case third is 1.29.the factor of safety of Aluminum 7075-T6 is greater than one .so the aluminum have ability to stand at higher load.

- Final conclusion of the analysis the Titanium Ti-6Al$4 \mathrm{~V}$ is the much batter material than other two materials Cast iron and titanium Ti-13V-11Cr-3Al. Because the yield stress of Titanium Ti-6Al-4V is higher the titanium Ti-13V-11Cr-3Al. And the density of the Titanium Ti-6Al-4V was lower than cast iron and titanium Ti-13V-11Cr-3Al.

\section{REFERENCES}

[1] M. M. Patunkar, Rameshwar SSAKHARAM Dolas "Modelling and Analysis of Composite Leaf Spring under the Static Load Condition by using FEA" January 2011.

[2] B. Pyttel, I. Brunnera "Fatigue behavior of helical compression springs at a very high number of cycles - Investigation of various influences" DOI: 10.1016/j.jifatigue.2013.01.003 March 2014.

[3] Yunan Prawoto, M. Ikeda "Design and failure modes of automotive suspension springs" DOI: 10.1016/j.engfailanal.2007.11.003 15(8):1155-1174 - December 2008.

[4] Ladislav Kosec, Aleš Nagode "Failure analysis of a motor-car coil spring” DOI: 10.1016/j.csefa.2013.12.004 4(C) · December 2013.

[5] Senthilkumar Mouleeswaran, S. Vijayarangan "Analytical and experimental studies on fatigue life prediction of steel and composite leaf spring for light passenger vehicles using life data analysis" Vol.13(No.2):pp.141-146 · June 2007.

[6] Bankole I. Oladapo, C.O. Ijagbemi "Design and Simulation of Fatigue Analysis for a Vehicle Suspension System (VSS) and its Effect on Global Warming” 10.1016/j.proeng.2016.08.135 .

[7] Izatul Hamimi Abdul Razak "Modeling, Simulation And Optimization Analysis Of Steering Knuckle Component For Race Car" DOI: 10.15623/ijret.2014.0311037 November 2014.

[8] Hari Dayalan, Yeshwant Raj.s "Investigation of Knuckle Joint and Its Importance -A Literature Case Study" January 2018. 
[9] Gaurav Saxena, Ankit Chauhan "Simulation and Optimization of wheel Hub and Upright of Vehicle: A Review" DOI: 10.9790/1684-1401034250 January 2017.

[10] Vi Kie Soo, Paul Compston, Paul Compston "Interaction between New Car Design and Recycling Impact on Life Cycle Assessment" DOI: 10.1016/j.procir.2015.02.055 December 2015. 